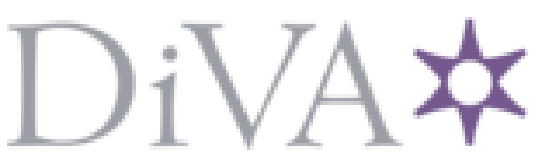

http://www.diva-portal.org

[This is not an article, chapter, of conference paper!] 


\title{
Estimating Attention in Exhibitions Using Wearable Cameras
}

\author{
Ali S. Razavian, Omid Aghazadeh, Josephine Sullivan and Stefan Carlsson \\ Computer Vision and Active Perception Laboratory \\ KTH, Sweden \\ Email: \{razavian,omida,sullivan,stefanc\}@kth.se
}

\begin{abstract}
This paper demonstrates a system for automatic detection of visual attention and identification of salient items at exhibitions (e.g. museum or an auction). The method is offline and is done on a video captured by a head mounted camera. Towards the estimation of attention, we define the notions of "saliency" and "interestingness" for an exhibition items. Our method is a combination of multiple state of the art techniques from different vision tasks such as tracking, image matching and retrieval. Many experiments are conducted to evaluate multiple aspects of our method. The method has proven to be robust to image blur, occlusion, truncation, and dimness. The experiments shows strong performance for the tasks of matching items, estimating focus frames and detecting salient and interesting items. This can be useful to the commercial vendors and museum curators and help them to understand which items are appealing more to the visitors.
\end{abstract}

\section{INTRODUCTION}

A commercial vendor, art or museum curator would like to know how long visitors pay attention to each item displayed in his gallery or exhibition. Currently, making such attention measurements requires specially designed systems (Tobii) with expensive and cumbersome hardware. However, the recent advent of small and affordable wearable cameras, such as the GoPro, and the mature field of geometric matching of visual images [1], make it possible to solve this attention problem automatically with low-cost hardware and standard computer vision algorithms. In this paper we exploit these hardware and software components to build an easy to use system which measures the amount of time a person, wearing a camera, looked at the items in an exhibition. We report the results when our system is applied to footage from several people visiting a recent photography exhibition in Stockholm, Sweden.

One of the main components of our system is to identify if an item is present in an image or not. Given a reference image of the item, a standard algorithmic pipeline exists to achieve this for a new frame [2] and also return the item's location if it is present. One simply extracts feature points and their descriptors in the two images, puts the two set of points in correspondence based on descriptor similarity and finally identifies the correct correspondences with your favourite version of RANSAC [3] to estimate the geometric transformation between them. This process works extremely well if the item is textured (lots of feature points) and planar (a simple homography ideally defines the transformation). Therefore our scenario of an exhibition of photographs and paintings is the ideal setting to exploit such a matching pipeline.

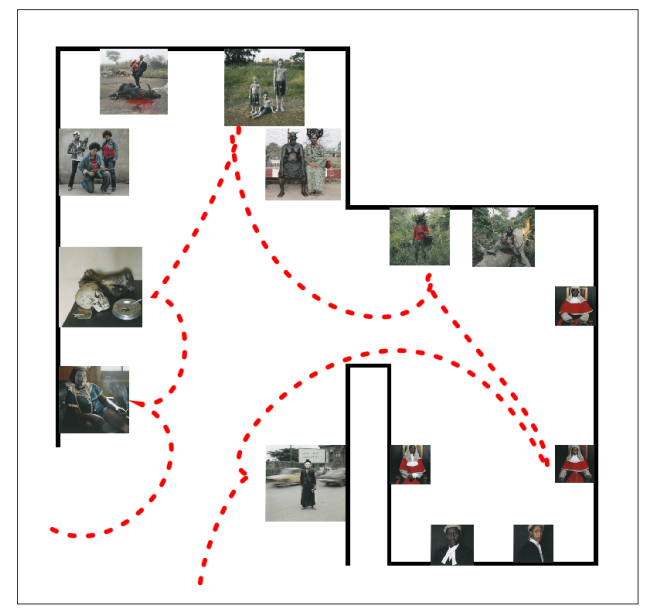

Fig. 1. A visitor wearaing a camera walks around an exhibition and stops at various items (colours) for differing lengths of time. An ideal system for summarizing exhibition attention would measure these attention spans for a selected number of people using the videos captured from the cameras. The area of each image is related to the length of time the item has been viewed.

It also means that we can focus on the particular computer vision problems associated with first person vision which as of yet do not have a standard solution. These are: How can we identify the frames when the camera wearer is paying attention to a referenced item? And how can we decide which item the person is actually looking at if there are multiple items in a frame? We now summarize how we solve these problems by giving an overview of the main elements of our system:

- Collect videos from a set of people walking around an exhibition wearing a small head mounted camera.

- From each video automatically extract consecutive frames that correspond to when the wearer is focused on an item. We perform this by looking for patterns, learnt in a supervised fashion, in the optical flow field induced by the motion of the camera wearer, section II-A. We refer to these sequences as focus shots.

- Locate, label and track the items throughout the focus shots, sections II-B and II-C.

- Frequently there are several items in a focus frame. Therefore we have to decide at which item the camera wearer is actually looking. We refer to this item as the salient item. The saliency of an item can be inferred by its position in the frames throughout a focus shot and its motion through the shot, section II-E. 


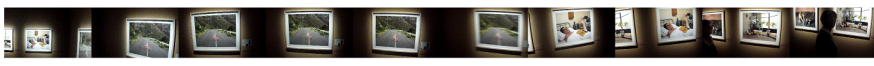

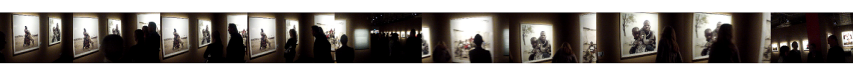

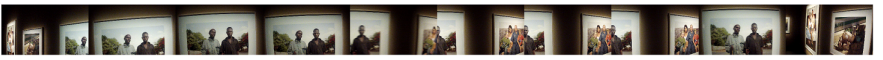

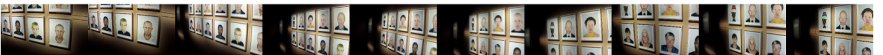

Fig. 2. 10 frames from 4 different shots of video subsampled at $0.5 \mathrm{~Hz}$

\section{METHOD}

Our work aims to identify interesting items in an exhibition from several videos taken by a head mounted wearable camera. We assume that interestingness of an item is correlated with the amount of time that people pay attention to it. Therefore, we aim to identify salient items in videos.

Our pipeline is as follow. First we use optical flow patterns to detect the frames when a person is paying attention to something. We call these frames focus frames. By grouping focus frames, we divide a video into focus shots and skip processing the rest of the video. From each focus shot we extract a keyframe and estimate each visible item's spatial boundary and track those boundaries throughout the entire video. Finally, from the tracked items we predict the salient item(s) in each focus shot.

\section{A. Focus Shot Detection}

Upon inspection of our captured video we realized that humans could easily and consistently recognize the frames when the camera wearer was paying attention to an item and those when he was looking around aimlessly. They also only needed short sequences to perform the recognition. We assumed then that it would be possible to infer the wearer's attention levels from the optical flow fields induced by the wearer's head movements. Therefore we train a linear SVM classifier to predict the binary label of focused/not focused when applied to an appropriate feature descriptor of frame $I_{t}$. We now describe the properties we want out feature to have and how we construct such a descriptor.

A good feature vector for our task should encode both the camera's velocity and acceleration. The feature vector should also model the rotation around the axes parallel to the image frame, but yet be invariant to rotation around perpendicular axis to the image plane.

We compute the optical flow field for each frame in the video. From these flow fields we obtain a set of point tracklets. Each point tracklet consists of a start and end frame and the point's image coordinates while it is tracked between these frames. We use these tracklets to create a feature descriptor for each frame $I_{t}$. The descriptor has the following elements: the number of point tracklets whose temporal extent includes both $I_{t}$ and $I_{t+n}$ for $n \in\{-N, \ldots, N\} \backslash\{0\}$; the min, max, mean, std and variance of the $x$ - and $y$-coordinates for all the tracklet points first in $I_{t}$ and then $I_{t+n}$ where the tracklets considered must contain both these two frames. These feature model the forward transition and angular velocity for $n \in\{1, \ldots, N\}$ and the reverse speed for $n \in\{-N, \ldots,-1\}$. In the same manner, we also compute the number, min, max, mean, std and variance for the tracklets containing the frames $I_{t-n}, I_{t}$, and $I_{t+n}$. These features model the acceleration of the camera. We aggregate all these features over the temporal neighborhood $N$ to compute a representation for $I_{t}$.

We normalize the feature vectors w.r.t. each dimension and then trained an $L_{1}$ normalized binary SVM classifier. At test time the output of the SVM classifier applied to each sequence is temporally smoothed and contiguous sequences of focus frames are grouped into focus shots. Our classifier proves to be quite robust, but the failures that do occur are triggered mainly by these three situations:

1) at the boundaries of focus shots.

2) when two items are very close to one another and the head's movement is smooth between them. In this case we expect to have two separate focus shots but instead the classifier usually predicts one focus shot.

3) when an item is larger than the camera's field of view and the head moves in a way that tracklets do not last through the entire shot. In this case we expect to have one focus shot but instead we get multiple shorter shots.

From our experiments we see that the head's movement for case 2 and 3 are usually very similar and it is almost impossible to correctly detect focus frames without any knowledge about the content of the frames.

\section{B. Retrieval}

We adopt standard retrieval pipelines for this part. We use the, current state-of-the-art, VLAD [4] encoding of the SIFT[5] descriptor as the representation of our feature points. Then we estimate the homography (our items are 2D planar objects) from top scoring correspondences between the reference and test frames. We considered three different scenarios of reference sets for this task.

- A catalogue containing high quality pictures in the exhibition is available.

- A catalogue in not available but it is possible to manually collect high resolution photos of the items in the exhibition. In this case, the photos may be blurry, occluded and/or noisy and this makes the matching and homography a slightly less robust process.

- There is no predefined catalogue and we annotate a small subset of the frames from the video and use these as our reference set of images.

In each reference image we also assume the bounding box of the item is annotated.

Estimating the homography between a frame and each reference image is a relatively expensive operation and it is not computationally feasible to estimate these homographies for every frame in each focus shot. Hence, we extract $M$ temporally equidistant keyframes from each focus shot and use these as query images for our retrieval system. After detecting the boundary for all the items in each keyframe, we track them between the keyframes using the pre-computed tracklets. We now describe how we compute these two process: the homography estimation and the tracking of the item boundaries between keyframes. 


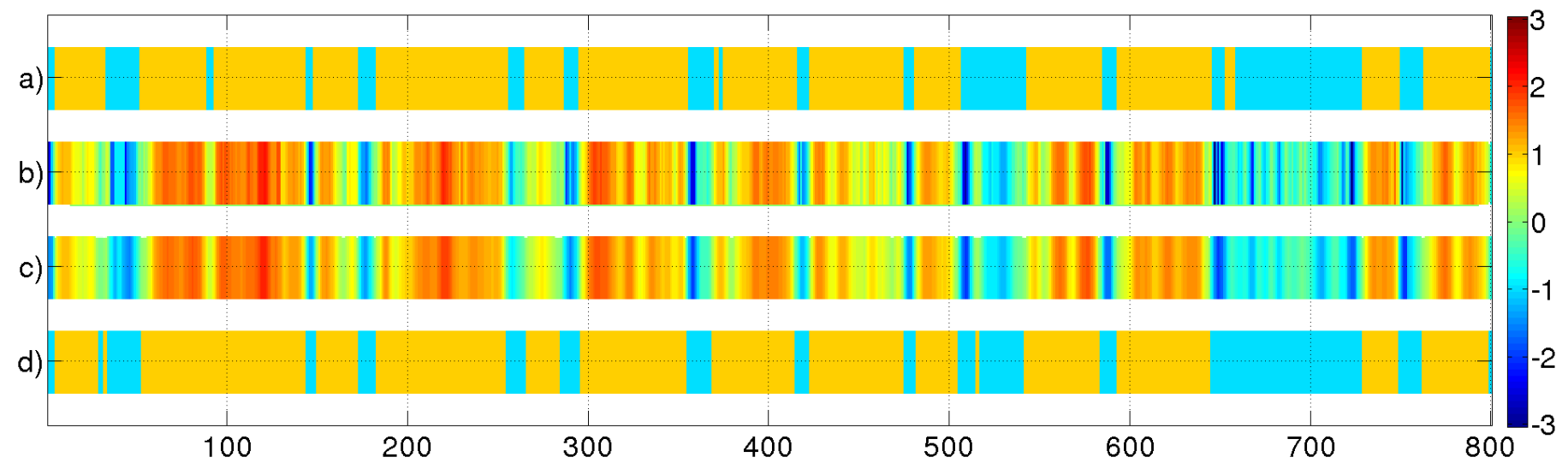

Fig. 3. Performance of focus frame detection in the first 800 frames of the test video compared to ground truth. Row a: Ground truth. Row b: Output of the focus frame classifier. Row c: Smoothed output using a moving average kernel of length 5. Row d: Binary smoothed output with threshold at 0. One can see the effectiveness of our focus shot detection by comparing row a and d. The difficulties usually arise when there are fast head motions to focus on different parts of the same item which are confused with moving from one item to another close item.

\section{Homography estimation}

To estimate the homography between a reference and query frame, we compute feature points independently in each frame and extract a feature descriptor for each point [5]. The points in the two frames are then put in correspondence based on the similarity of these feature descriptors. Only some of the computed correspondences will be correct. Initially, we prune the large set of correspondences by removing those whose feature descriptors are not discriminative as suggested by [5]. We further prune the set by analyzing the differences between the scale and rotation of corresponding feature points.

In detail let $\left(s_{i}^{(r)}, \theta_{i}^{(r)}\right)$ be the scale and rotation of the $i^{t h}$ feature point found in the reference image and let $\left(s_{j}^{(q)}, \theta_{j}^{(q)}\right)$ represent the same quantity for the $j^{\text {th }}$ query image. Then let $\left\{\left(i_{l}, j_{l}\right)\right\}_{l=1}^{L}$ be the indices of the feature points in correspondence. As we are interested in correspondences located on paintings/photographs and items' planes are approximately parallel to the image plane we do not expect the rotations of these corresponding points to differ significantly. Therefore we only retain correspondences $\left(i_{l}, j_{l}\right)$ s.t.

$$
-\theta \leq \theta_{i_{l}}^{(r)}-\theta_{j_{l}}^{(q)} \leq \theta
$$

Next adopting the idea of [6] we project the correspondences into Scale-Rotation space

$$
\left(\log \left(\frac{s_{i_{l}}^{(r)}}{s_{j_{l}}^{(q)}}\right), \theta_{i_{l}}^{(r)}-\theta_{j_{l}}^{(q)}\right) .
$$

Then we grid this space and count the number of correspondences in each grid cell. We prune every pair that lies in a cell with a population less than $p$. After this pruning, the ratio of inliers to outliers increases drastically.

The bounding region of the items are known in the reference images. We therefore employ PROSAC [7] over the remaining correspondence that lie inside the item's boundary and estimate the homography matrix. For each item $O_{i}$ in the reference image $I_{r}$ we pick $K$ homography matrices which result in the most number of inliers. We use each homography $H_{r \rightarrow q}^{i}$ from the reference image to the query image, $I_{q}$, to

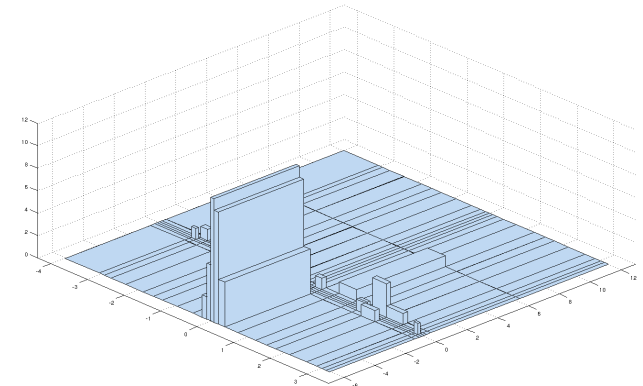

Fig. 4. Histogram of the correspondences in Scale-Rotation space. While inlier correspondences tend to cluster in this space, outliers are uniformly distributed.

transfer boundary of $O_{i}$ to $I_{q}$ and also to define a confidence measure for the transferred boundary $B_{r \rightarrow q}^{i}$ with:

$$
\operatorname{Conf}\left(B_{r \rightarrow q}^{i}\right)=\max \left(0,\left(1-e^{-\left(n_{\text {inliers } H_{r \rightarrow q}^{i}}-n_{0}\right)}\right)^{\alpha}\right)
$$

where $n_{0}$ is the minimum number of inlier correspondences.

\section{Tracking}

After detecting each item's boundary in all the keyframes, we track these boundaries in each shot using tracklets. In order to annotate frame $I_{q+t}$, we pick tracklets inside $B_{r \rightarrow q}^{i}$ in keyframe $I_{q}$ which survive to frame $I_{q+t}$. The tracklets allow us to track feature points from $I_{q}$ to $I_{q+t}$ and then, of course, compute the homography matrix between item $O_{i}$ in frames $I_{q}$ and $I_{q+t}$. We define the confidence for this boundary as:

$\operatorname{Conf}\left(B_{r \rightarrow q \rightarrow(q+t)}^{i}\right)=\left(1-e^{-|t|}\right)^{\beta} \operatorname{Conf}\left(B_{r \rightarrow q}^{i}\right) \operatorname{Conf}\left(B_{q \rightarrow q+t}^{i}\right)$

Finally, we compute our confidence for every $O_{i}$ in every frame $I_{f}$ as the sum over confidence values of all the reference images containing $O_{i}$ and all keyframes $I_{q}$ in the focus shot containing frame $I_{f}$ :

$$
\operatorname{Conf}\left(B_{f}^{i}\right)=\min \left(1, \sum_{r, q} \operatorname{Conf}\left(B_{r \rightarrow q \rightarrow f}^{i}\right)\right)
$$



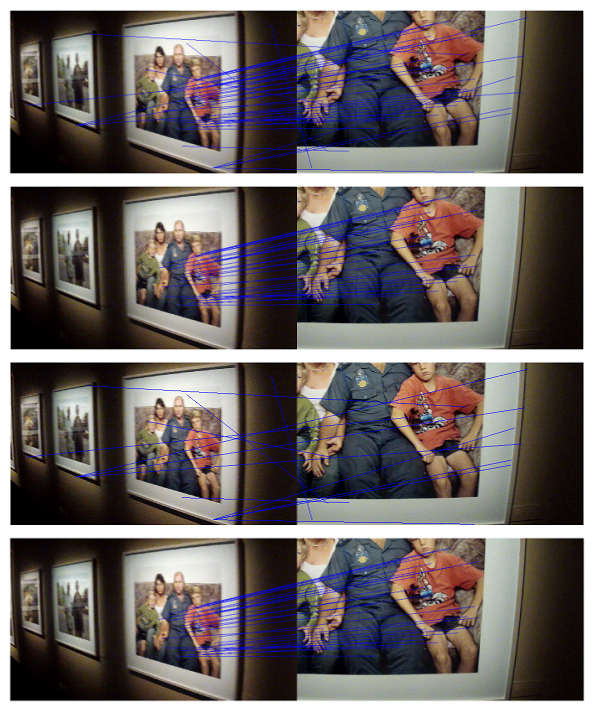

Fig. 5. Correspondences between two key frames from the dataset. Row 1: All the distinctive correspondences. The ratio of inliers to number of correspondences is 0.71 . Row 2: Distinctive correspondences that agree in Scale-Rotation space, inlier ratio $=1$. Row 3: Distinctive correspondences pruned by the Scale-Rotation space. Row 4: The inliers w.r.t. homography.

If $\operatorname{Conf}\left(B_{f}^{i}\right)$ is bigger than a threshold $\sigma$ we set the boundary of $O_{i}$ in $I_{f}$ where the summations are over the same quantities just described:

$$
B_{f}^{i}=\frac{\sum_{r, q} \operatorname{Conf}\left(B_{r \rightarrow q \rightarrow f}^{i}\right) B_{r \rightarrow q \rightarrow f}^{i}}{\sum_{r, q} \operatorname{Conf}\left(B_{r \rightarrow q \rightarrow f}^{i}\right)}
$$

In the description above, we assumed there is only one homography computed for $O_{i}$ in reference image $I_{r}$ to each query image $I_{q}$. However $K$ such homographies are estimated. PROSAC predicts multiple homographies and we chose the top $K$ w.r.t. the number of inliers. Therefore, to build a more robust system, we update the confidence of an item boundary $B_{f}^{i}$ to:

$$
\operatorname{Conf}\left(B_{f}^{i}\right)=\sum_{k=1}^{K} \operatorname{Conf}\left(B_{f}^{i, k}\right)
$$

and

$$
B_{f}^{i}=\frac{\sum_{k} \operatorname{Conf}\left(B_{f}^{i, k}\right) B_{f}^{i, k}}{\operatorname{Conf}\left(B_{f}^{i}\right)}
$$

where $\operatorname{Conf}\left(B_{f}^{i, k}\right)$ and $B_{f}^{i, k}$ represent the confidence and boundary found with the $k^{t h}$ homography respectively.

\section{E. Salient Item Detection}

Human subjects, most of the time, agree on the salient item in a frame just by watching a focus shot. Here we assume the salient item is the item in the frame that the person is looking at which implies the saliency of an item is related to its relative position in the image over time. We employ a supervised approach to learn this tempo-spatial property. First, we create a feature vector for each item $O_{i}$ in every focus frame $I_{t}$. This feature vector consists of the concatenation of the following simple features:

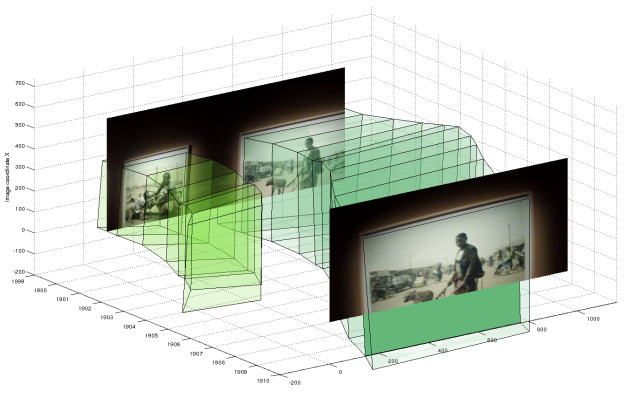

Fig. 6. Tracking an item's boundary in a video. Frame 1899 of the test video transfers its annotation to frames 1900 to 1910

- $\quad$ position of $O_{i}$ in $I_{t}$.

- position of $O_{i}$ in $I_{t}$ substracted from position of $O_{i}$ in $I_{t+n}$ for temporal neighborhood $n \in$ $\{-N, \ldots, N\} \backslash\{0\}$. The position is a vector of length $2 m$ containing the image coordinates of the $m$ boundary points of item $O_{i}$.

- the area of $O_{i}$ in $I_{t}$.

- the area of $O_{i}$ in $I_{t}$ substracted from the area of $O_{i}$ in $I_{t+n}$. for temporal neighborhood $n \in$ $\{-N, \ldots, N\} \backslash\{0\}$.

- $\quad$ ratio of $O_{i}$ that lies inside $I_{t}$.

- $\quad$ ratio of $I_{t}$ that covers $O_{i}$.

- a binary feature indicating whether this item is the only item in this frame or not.

- the distance from the center of the frame to the closest point on the item's boundary.

We normalized the feature vectors w.r.t. each dimension and then trained an $L_{1}$ normalized binary SVM classifier using liblinear [8]. Then for each item we apply an averaging kernel of temporal length $T$ over the SVM predictions. Finally, for each frame we pick up the maximum predictions above a threshold $\theta$ if they exist.

\section{EXPERIMENTS}

In this section we report the results of applying our system to the dataset we created. To begin we give an overview of our dataset.

\section{A. Dataset}

We chose Pieter Hugo's exhibition "This must be the place" in the Fotografiska museum of photography. We used the GoPro Hero2 camera to capture 5 videos of resolution $1980 \times 1020$. In total we captured 62 minutes of footage at 30 frames per second. We sub-sampled this footage at $5 \mathrm{~Hz}$ and resized these 18776 images to $960 \times 540$. We annotated the dataset with 3 levels of information:

- $\quad$ is the person paying attention to something or not.

- which item is salient.

- boundaries of the items in every frame. 


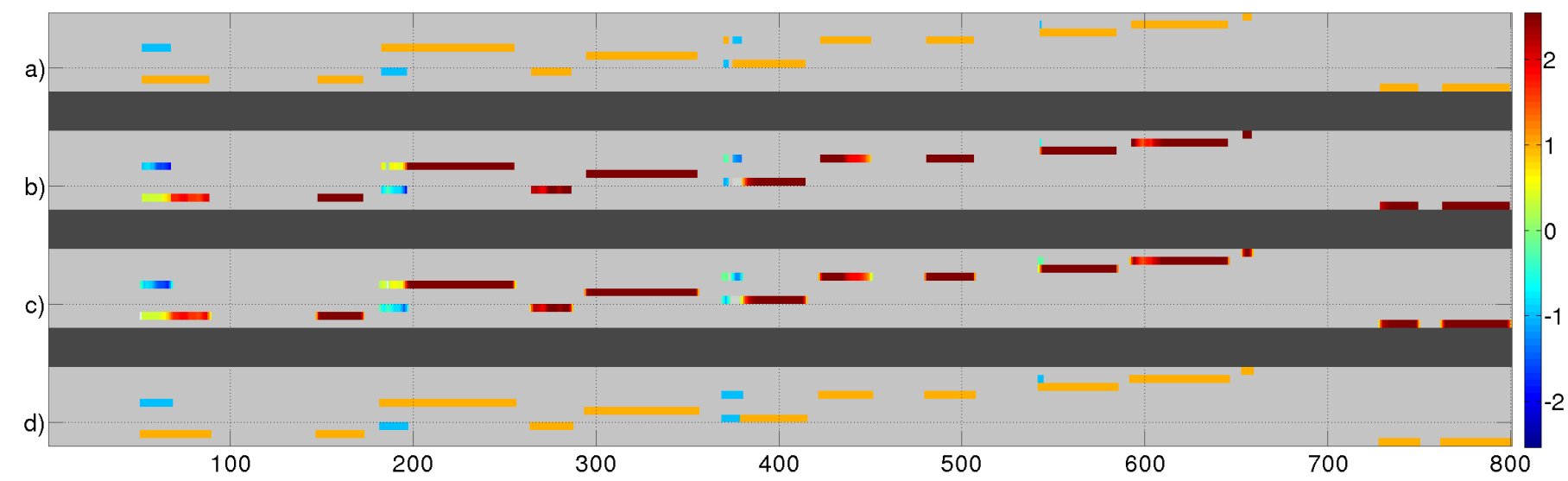

Fig. 7. Salient item predictions for the first 800 frames of the test video (only for the 10 items appearing in these 800 frames). A negative value (blue for binary case) indicates a non-salient item and a positive value (orange for binary case) indicates a salient item. Refer to the scale color bar for other colors. Row a: Ground truth. Row b: Response of the salient item classifier. Row c: The smoothed responses using a moving average kernel of length 5. Row d: The thresholded smoothed responses with threshold set to 0 .

There are 380 focus shots in these videos with 108 distinct items. Among them, 73 have been annotated as salient. On average, there are $1.38 \pm 1.08$ items in each frame. On the other hand, each item has appeared in $239.90 \pm 122.47$ frames. We used 3 videos (179 focus shots, 9283 frames) for training, one (100 focus shots, 4660 frames) for cross validation and one for test (101 focus shots, 4833 frames). Our dataset is publicly available at FILL THIS IN.

\section{B. Focus Shot Detection}

We used the publicly available code of [9] to compute the optical flow fields with a sampling step of 10?? (over resized images $540 \times 403$ for computational efficiency). We computed the feature vector for each frame from a temporal window of 1 second. We used liblinear [8] to train the binary SVM focus frame classifiers. The trained classifier achieves an accuracy of $\mathbf{9 0 . 2 1} \%$ on the test frames and $90.97 \%$ for all the frames. The small difference between the performance on the train and test set indicates low the variance of the final classifier (less overfitting) while maintaining low bias (training error). The accuracy is measured as the ratio of the number of correctly classified feature vectors to all vectors. The grouping of the half-wave rectified frame scores generates 129 focus shots for the test video and 500 focus shots for the entire video. A moving average kernel of size 5 results in a focus frame detection accuracy of $\mathbf{9 0 . 0 2} \%$ for the test video and $91.17 \%$ for all videos. However, the number of focus shots was decreased to 91 shots on the test video and 355 shots on all videos. This shows while the average kernel has little effect on the accuracy of focus frame detections, it helps to reduce the number of distinct focus shots by removing noise from focus frame prediction. Figure 3 shows the performance of each of these steps compared to the ground truth. One can see the effectiveness of our focus shot detection by comparing rows (a) and (d). Difficulties usually arise when there are fast head motions to focus on different parts of the same item, these motions are confused with moving from one item to another close item.
TABLE I. F-MEASURE FOR THE RETRIEVAL TASK MEASURED USING DIFFERENT REFERENCE SETS WITH VARIOUS NOISE LEVELS.

\begin{tabular}{lc}
\hline Reference set & F-measure \\
\hline Clean catalogue & $95.21 \%$ \\
Noisy catalogue & $94.57 \%$ \\
Key frames & $94.62 \%$ \\
\hline
\end{tabular}

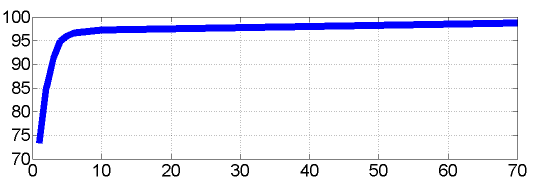

Fig. 8. F-measure for our retrieval system, the reference set is the clean catalogue, computed on a validation set as the number of keyframes extracted from a focus shot varies. Performance saturates at around 6 keyframes.

\section{Retrieval}

We use the F-measure to evaluate the performance of our retrieval system. We divided the Scale-Rotation space into a grid of $8 \times 25$ regions and treated all correspondences that lie alone in a grid cell $(p=1)$ or do not satisfy $-\pi / 4 \leq \theta_{i_{l}}^{(r)}-\theta_{j_{l}}^{(q)} \leq \pi / 4$ as outliers. We chose the top $K=15$ homography matrices between every match and set the confidence parameters $\beta=0.2$ and $\alpha=0.2$. We set the threshold $\sigma=0.1$ as it maximized the F-measure value during cross validation. We only counted annotations inside focus shots and ignored the rest. We only count an item boundary as a true positive if its predicted boundary and annotated boundary overlap more than $50 \%$. The overlap is measured as the area of their intersection over the area of their union. We chose 6 key frames per focus shots since the increase in F-measure for more than 6 frames was negligible (Figure 8). Table I shows the F-measure for difference cases of a clean reference set (catalogue), noisy reference set (manually captured pictures) and training set annotated key-frames. The results show one can achieve robust results from minimal annotation effort (only a few keyframes of one video). This is probably due to the fact that the matching uses several frames of a shot produced by our tracking and this reduces the effect of noisy keyframes. 


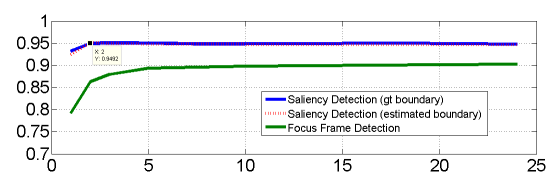

Fig. 9. The effect of the temporal neighborhood size on both focus and salient item detection. The accuracy of the saliency predictions saturates for temporal neighborhoods greater than $\pm 1 \mathrm{sec}$. On the other hand, the accuracy of focus predictions keeps increasing.

TABLE II. F-MEASURE FOR SALIENT ITEM DETECTION

\begin{tabular}{lllc}
\hline Attention labels & Item(s) annotation & Reference set & F-measure \\
\hline Ground truth & Ground truth & - & $88.68 \%$ \\
Ground truth & Predicted & clean catalogue & $87.99 \%$ \\
Ground truth & Predicted & noisy catalogue & $87.26 \%$ \\
Ground truth & Predicted & key frames & $87.52 \%$ \\
Predicted & Predicted & clean catalogue & $84.18 \%$ \\
Predicted & Predicted & noisy catalogue & $83.20 \%$ \\
Predicted & Predicted & key frames & $82.75 \%$ \\
\hline
\end{tabular}

\section{Salient Item Detection}

We used 2-fold cross validation to find the best average kernel length of 3 for all experiments. We evaluated different choices for the $C$ parameter (SVM misclassification cost) and we fixed it to $C=0.01$ for a temporal range of \pm 1 second. The accuracy of detecting these salient item of test frames is $95.1 \%$ for the case where the boundaries comes from the ground truth and $95.0 \%$ when we estimate the boundary. The negligible difference indicates the high quality of item boundary detection. Figure 9 shows the accuracy against different sizes of temporal neighborhood. The performance does not increase by increasing the neighborhood length after 1 second for saliency detection but focus frame detection saturates later. This effect is because at this level (saliency detection) we have more evidence of the current frame and need less information from the neighborhood.

For evaluation purpose, we labelled each frame to its predicted salient item index. Then we computed F-measure with internal parameter $\beta=1$ to evaluate the performance of item saliency detection. We computed the histogram for the time each item has been in center of attention and intersected the result with histogram of ground truth. Table II has the evaluated F-measure for 7 different scenarios arising from altering the source of annotation for focus labels and item boundaries.

Our final goal was to estimate the "interesting" items by measuring the amount of time visitors spend looking at them. For that we generate a feature vector with its $i$ th dimension referring to the total time that all visitors have spent focusing on item $i$. We call this vector the interestingness vector. To evaluate the performance in this regard, we also generate the ground truth interestingness vector from annotations and use histogram intersection similarity measure on the $L_{1}$ normalized interestingness vector. This gives a similarity rate of 0.98 on the clean catalogue reference set, 0.97 for the noisy catalogue reference set and 0.98 for key frames used as the reference set. The high similarity of the two vectors indicate the effectiveness of our complete method in reporting interesting items. Refer to figure 10 for an illustration of the two vectors.

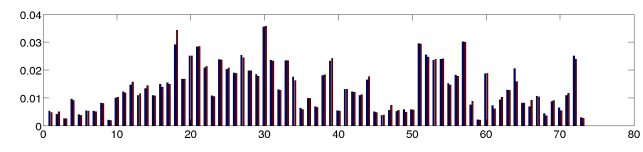

Fig. 10. $L_{1}$ normalized interestingness vectors estimated from the ground truth (blue) and the method's output (red). The $i$ th dimension of the interestingness vector refers to the total time that all visitors have spent focusing on item $i$.

\section{CONCLUSION}

In this work, we have presented an effective combination of the state-of-the-art methods for the task of estimating visitors' attention at an exhibition. To achieve this we defined, learned and estimated some relevant notions such as the "interestingness" and "saliency" of an item. These notions could help the design and maintenance of such exhibitions.

We evaluated every part of the system with different measures and showed its high accuracy and robustness to different scenarios. Most importantly we have shown that one can effectively estimate the "interestingness" of items in an exhibition to a high degree by using our system.

Through the experiments we demonstrated several indicators of the potential to decrease the annotation by a large degree and yet be effective enough for estimation of different properties.

One possible line of future work is to try the method on $3 \mathrm{D}$ objects with less textures (such as sculptures). This will make the matching of items a more challenging task as it will involve estimation of the $F$ matrix instead of homographies and needs more robust features in the absence of textures. Another interesting direction is the case where there is no prior knowledge about the content of the exhibition, which will turn all the recognition (matching) tasks into unsupervised clustering. Furthermore, there are cases where the visitor can have interactions with the items of exhibition (e.g. silent auction) which will make the task rather more difficult.

\section{REFERENCES}

[1] M. Brown and D. Lowe, "Automatic panoramic image stitching using invariant features," International Journal of Computer Vision, vol. 74, no. $1,2007$.

[2] J. Sivic and A. Zisserman, "Video google: A text retrieval approach to object matching in videos," in ICCV, 2003, pp. 1470-1477.

[3] M. Fischler and R. Bolles, "Random sample consensus: A paradigm for model fitting with applications to image analysis and automated cartography," Communications of the ACM, vol. 24, no. 6, 1981.

[4] R. Arandjelović and A. Zisserman, "All about VLAD," in Proceedings of the Conference on Computer Vision and Pattern Recognition, 2013.

[5] D. Lowe, "Distinctive image features from scale-invariant keypoints," International Journal of Computer Vision, vol. 60, no. 2, 2004.

[6] M. Brown and D. G. Lowe, "Invariant features from interest point groups," in Proceedings of the British Machine Vision Conference, 2002.

[7] O. Chum and J. Matas, "Matching with PROSAC - progressive sample consensus," in Proceedings of the Conference on Computer Vision and Pattern Recognition, 2005.

[8] R.-E. Fan, K.-W. Chang, C.-J. Hsieh, X.-R. Wang, and C.-J. Lin, "LIBLINEAR: A library for large linear classification," Journal of Machine Learning Research, vol. 9, pp. 1871-1874, 2008.

[9] N. Sundaram, T. Brox, and K. Keutzer, "Dense point trajectories by gpu-accelerated large displacement optical flow," in Proceedings of the European Conference on Computer Vision, 2010. 